\title{
Vulnerability in light of the COVID-19 crisis
}

\author{
Henk ten Have ${ }^{1} \cdot$ Bert Gordijn ${ }^{1}$
}

Published online: 1 April 2021

(c) The Author(s), under exclusive licence to Springer Nature B.V. 2021

The Covid-19 pandemic clearly illustrates the various dimensions of the notion of vulnerability. Human beings in general are vulnerable, because their bodies are embedded in environments that can harm them. Everybody can be affected by viruses because micro-organisms are essential components of the biosphere and necessary for the sustenance of life. Special vulnerability exists for human beings that are more susceptible to harm than others. The pandemic demonstrates that healthcare workers are more frequently infected, because of their increased exposure. In addition, several other groups are more at risk for different reasons: older people, persons with underlying conditions or compromised immune systems, socio-economically disadvantaged people, and racial and ethnic minorities.

Overall, general as well as special vulnerability have increased through processes of globalization that have resulted in a world that creates more and new threats. At the same time, these processes have undermined traditional protection mechanisms such as social security and welfare systems, and family support mechanisms, thus eroding the abilities of individuals and communities to cope with serious threats. The fact that the world has become increasingly interconnected and interdependent has created a sense of mutual vulnerability. Being vulnerable is often the result of a range of social, economic and political conditions, and therefore beyond the power and control of individuals.

Because of this, vulnerability can no longer be framed, as it usually is in mainstream bioethics, as an individual affair. This traditional understanding is informed by the principle of respect for autonomy. It is assumed that fragility or weakness impair the capacity of persons to make autonomous decisions (for example, the capability to give informed consent). As a result, they no longer have the ability to protect their own interests and need special protection. From the perspective of global bioethics, however, vulnerability is

Henk ten Have

tenhaveh@duq.edu

1 Leiden, The Netherlands analyzed as caused by structural social, economic and political determinants that disadvantage people. This analysis of vulnerability as a global phenomenon refers to the ethical principles of justice, solidarity and equality, rather than individual autonomy and has two important implications for the bioethical debate.

The first is the recognition that humans are social beings. The common idea that individual persons are autonomous and in control is challenged. Since the human condition is inherently fragile, all human beings are sharing the same predicament. Because our bodily existence is vulnerable, and embedded in environments such as the virosphere, humans have developed institutions and social arrangements to protect themselves. Vulnerability exists because human beings are open to the world, they engage in relationships with other persons, and interact continuously with the world. This openness and interaction is a positive phenomenon; it is the basis for exchange and reciprocity between human beings. We cannot come into being, flourish and survive if our existence is not connected to the existence and flourishing of others. The notion of vulnerability therefore refers to solidarity and mutuality, the needs of groups and communities, not just those of individuals. In this perspective, vulnerability is neither an individual accomplishment or deficit.

The second implication is that vulnerability should mobilise a different response: if vulnerability is a symptom of the growing precariousness of human existence and is exacerbated under certain conditions, the social and environmental context can no longer be ignored in bioethical analysis. On the contrary, bioethics should focus on the distribution and allocation of vulnerability at global level. Instead of focusing on individual deficits, bioethical analysis should criticise the external determinants that expose individuals to possible damage and harm. It also means that individual responses are insufficient; what is needed is a collective response, in other words social and political action. However, such a collective response should have a global outreach. While many countries implement their own policies, often not harmonized or coherent with those of other countries, vulnerability at the global level will continue to exist, and this will 
prolong vulnerabilities at national levels. Current vaccination strategies illustrate this point as often articulated by the World Health Organization: Nobody is safe until everybody is safe.

In this issue, Janet Delgado (2021) extends the notion of vulnerability to the debate on professionalism. She argues that this debate is often limited to individual competencies of practitioners combining knowledge, know-how, and ethical sensitivity. Recognition that healthcare professionals are vulnerable as all human beings shifts the focus of professionalism in two important ways.

First, it emphasizes the significance of relationships and relationality in healthcare practices. Working in healthcare implies openness to other people in difficult circumstances of their lives, and thus a constant confrontation with death, suffering and pain. Healthcare professionals are more than other people exposed to tragic and burdensome experiences that may lead to moral distress and burnout. This has become especially clear in the Covid-19 pandemic revealing the vulnerability of healthcare workers, not so much in the sense that they have a higher risk to be infected but that they have to cope with a sometimes excessive burden of caring for very ill and dying patients.

Second, the shift in focus acknowledges not only the vulnerability of healthcare professionals but also that of patients and healthcare institutions. Because of the important role of relationality, these vulnerabilities are connected and shared. The vulnerability of patient, as Delgado argues, is not only depending on their illness but even more so on the relationship with caring professionals. She furthermore argues that healthcare institutions have an important role to play in addressing these shared vulnerabilities through creating an organizational culture that enhances resilience in professionals and patients.

What she does not discuss, however, is the fact that institutions themselves can be vulnerable. One of the basic fears in the Covid-19 pandemic is that healthcare institutions might collapse. In most countries they have to learn how to be more resilient and able to cope with the surge of infectious cases, sometimes in the hard way, being unable to provide sufficient protection to care workers, having insufficient testing capacities, triaging patients for intensive care, and descaling care services necessary for non-Covid patients. Since professionalism is not simply an individual affair but depends on the institutional and social setting in which it operates, it will be unavoidable to protect the vulnerability of healthcare institutions themselves.

\section{Reference}

Delgado, J. 2021. Vulnerability as a key concept in relational patientcentered professionalism. Medicine Health Care and Philosophy. https://doi.org/10.1007/s11019-020-09995-8.

Publisher's Note Springer Nature remains neutral with regard to jurisdictional claims in published maps and institutional affiliations. 